\title{
ON THE EXISTENCE OF MINIMAL IDEALS IN A BANACH ALGEBRA
}

\author{
BY \\ BRUCE A. BARNES
}

Introduction. In this paper we give a sufficient condition that an algebra have a minimal left (or right) ideal. Specifically, we prove that if $A$ is a complex semisimple Banach algebra with the property that the spectrum of every element in $A$ is at most countable, then $A$ has a minimal left ideal. If $A$ is an $A^{*}$-algebra, we prove that $A$ has a minimal left ideal if the spectrum of every self-adjoint element of $A$ is at most countable. These two basic results are given in $\$ 2$, and some applications of them are given in $\$ 3$ and 4. The main result of $\S 3$ is Theorem 3.1 which is a variant of a theorem concerning $B^{*}$-algebras that was proved through the separate efforts of M. A. Naĭmark [5] and A. Rosenberg [7]. In $\$ 4$ we prove that a complex semisimple Banach algebra with the property that the spectrum of every element in the algebra has no nonzero limit points is a modular annihilator algebra. This together with a previous result of the author shows that modular annihilator Banach algebras are characterized by this property.

1. Notation. We deal exclusively with complex algebras $A$. The norm of an element $u \in A$ will be denoted by the usual symbol $\|u\| . \sigma_{A}(u)$ is the spectrum of $u$ in the algebra $A$. If $A$ is commutative, $u^{\prime}$ denotes the image of $u \in A$ by the Gelfand representation of $A$ on its maximal ideal space. We write the radical of $A$ as $\operatorname{rad}(A)$ and the socle of $A$ as $S_{A}$. If $E$ is any nonempty subset of $A$, then $L[E]$ and $R[E]$ are the left and right annihilators of $E$ respectively $(L[E]=\{a \in A \mid a E=0\})$. When $A$ has an involution *, then $u \in A$ is self-adjoint when $u=u^{*} . A$ is an $A^{*}$-algebra if there is a norm $|\cdot|$ on $A$ (not necessarily complete) with the property that $\left|u u^{*}\right|$ $=|u|^{2}$ for all $u \in A$. If $A$ is complete in this norm, $A$ is a $B^{*}$-algebra. Definitions of most of the concepts mentioned in this paper may be found in C. Rickart's book [6] (although our notations often differ). Information concerning modular annihilator algebras may be found in [1] or [8].

2. The existence of minimal ideals. We first treat the special case where the algebra $A$ is commutative in Theorem 2.1. Using this special case, we give the general result in Theorem 2.2. Finally in Theorem 2.3 we consider the case where $A$ is an $A^{*}$-algebra.

THEOREM 2.1. Assume that $A$ is a semisimple commutative Banach algebra with no minimal ideals. Then there is an element in A with uncountable spectrum.

Received by the editors January 1, 1967 and, in revised form, April 26, 1967. 
Proof. We assume for convenience that $A$ has an identity. Let $\Omega$ be the maximal ideal space of $A$. First suppose that there is a connected subset of $\Omega, \Gamma$, which contains more than one point. Then there exists $v \in A$ such that $v^{\prime}$ is not constant on $\Gamma$. But then $v^{\prime}(\Gamma)$ is a connected subset of the complex plane which contains more than one point. It follows that $v^{\prime}(\Gamma)$, and hence $\sigma_{A}(v)$, is uncountable.

In the remaining case every nonempty connected subset of $\Omega$ must consist of a single point, that is $\Omega$ is totally disconnected. If $\Omega$ contains an isolated point $\phi$, then by Silov's Theorem [6, Theorem (3.6.3), p. 168] there exists $e \in A$ such that $e^{\prime}(\phi)=1$, and $e^{\prime}(\psi)=0$ when $\psi \in \Omega, \psi \neq \phi$. Then $A e$ is a minimal ideal of $A$ which is contrary to hypothesis. Thus $\Omega$ contains no isolated points, and this implies that every nonempty open subset of $\Omega$ is infinite.

Now under the assumptions that $\Omega$ is totally disconnected (t.d.) and that every nonempty open and closed subset of $\Omega$ is infinite, we construct an element of $A$ which has uncountable spectrum. Since $\Omega$ is t.d., we may choose mutually disjoint, nonempty, open and closed subsets of $\Omega, V_{0}$ and $V_{1}$ such that $\Omega=V_{0} \cup V_{1}$. The sets $V_{0}$ and $V_{1}$ are t.d. and infinite, so we may choose as before mutually disjoint, nonempty, open and closed subsets of $V_{k}, V_{k 0}$ and $V_{k 1}$, such that $V_{k}=V_{k 0} \cup V_{k 1}$, $k=0,1$. In general by this same procedure, given $V_{k_{1} k_{2} \cdots k_{n}}, k_{j}=0$ or 1 , we may choose mutually disjoint, nonempty, open and closed subsets of this set, $V_{k_{1}} \cdots k_{n} 0$ and $V_{k_{1} \cdots k_{n} 1}$, such that $V_{k_{1} \cdots k_{n}}=V_{k_{1} \cdots k_{n} 0} \cup V_{k_{1} \cdots k_{n} 1}$. By this construction we obtain a collection of nonempty open and closed subsets of $\Omega,\left\{V_{k_{1} k_{2}} \cdots k_{m}\right\}$ where $k_{j}=0$ or 1 and $1 \leqq m<\infty$. Let $e_{k_{1} k_{2} \cdots k_{m}}$ be the idempotent in $A$ such that $e_{k_{1} k_{2} \cdots k_{m}}^{\prime}$ is the characteristic function of the set $V_{k_{1} k_{2}} \cdots k_{m}$ (such an idempotent exists in $A$ by Silov's Theorem).

Let $t_{1}=e_{1}$, and choose an integer $m_{1} \geqq 1$ such that $\left\|10^{-m_{1}} t_{1}\right\| \leqq 1$. Let $t_{2}=e_{01}+e_{11}$,

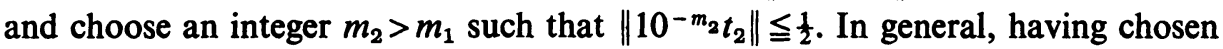
$m_{n-1}$, let

$$
t_{n}=\left(\sum_{k_{1}=0}^{1}-\sum_{k_{2}=0}^{1} \ldots \sum_{k_{n}=1=0}^{1}\left(e_{k_{1} k_{2} \cdots k_{n-1} 1}\right)\right),
$$

and choose an integer $m_{n}>m_{n-1}$ such that $\left\|10^{-m_{n}} t_{n}\right\| \leqq\left(\frac{1}{2}\right)^{n-1}$. Finally define $u=\sum_{k=1}^{\infty} 10^{-m_{k}} t_{k}$ (the sum converges in $A$ by the construction).

Now given any sequence of the digits 0 and $1,\left\{j_{1}, j_{2}, j_{3}, \ldots\right\}$, the set $\bigcap_{n=1}^{\infty} V_{j_{1} j_{2} \cdots j_{n}}$ is nonempty by the finite intersection property. Take a point $\phi$ in this set. Then $u^{\prime}(\phi)=\sum_{k=1}^{\infty} j_{k} 10^{-m_{k}}$. But the set of all such points is uncountable, and thus $\sigma_{A}(u)$ is uncountable.

THEOREM 2.2. Assume that $A$ is a semisimple Banach algebra with the property that the spectrum of every element of $A$ is at most countable. Then $A$ has minimal left and right ideals, and also $L\left[S_{A}\right]=0$.

Proof. We may exclude the trivial case when $A$ is $0 . A$ is semisimple, and for any $u \in A$ with nonzero spectrum, $\sigma_{A}(u)$ must have an isolated point. Thus $A$ contains a 
nonzero idempotent; see [4, Theorem 5.5.2, p. 175]. Let $E$ be a maximal set of nonzero commuting idempotents of $A$. Let $C$ be a maximal commutative subalgebra of $A$ containing $E$, and denote the space of modular maximal ideals of $C$ by $\Omega$. Now the algebra $C / \operatorname{rad}(C)$ is a semisimple commutative Banach algebra with the property that every element has at most a countable spectrum. By Theorem 2.1, $C / \mathrm{rad}(C)$ has a minimal ideal, or what is equivalent, $\Omega$ has an isolated point $\phi$. Again by Silov's Theorem [6, Theorem (3.6.3), p. 168] there is an idempotent $e \in C$ such that $e^{\prime}(\phi)=1$ and $e^{\prime}(\psi)=0$ when $\psi \in \Omega, \psi \neq \phi$. Assume that $g \in E$. Then for any $\psi \in \Omega,\left(g^{\prime}(\phi) e-g e\right)^{\prime}(\psi)=0$. Thus $g^{\prime}(\phi) e-g e \in \operatorname{rad}(C)$. Now $g^{\prime}(\phi)=0$ or 1 , and in either case $g^{\prime}(\phi) e-g e$ is an idempotent and is in $\operatorname{rad}(C)$. But the radical of any algebra contains no nonzero idempotents, and therefore $g^{\prime}(\phi) e=g e=e g$.

Consider the semisimple algebra $B=e A e$. Suppose that $f$ is a nonzero idempotent in $B$. Then $f=e f=f e$. If $g \in E$, then $f g=f e g=g^{\prime}(\phi) f e=g^{\prime}(\phi) e f=g f$. Therefore by the definition of $\mathrm{E}, f \in E$. It follows that $e f=f e=e$, and since $f e=f$, we have $f=e$. We have shown that $e$ is the only nonzero idempotent in $B$. It follows by [4, Theorem 5.5 .2$, p. 175] that the spectrum of each element of $B$ is connected. Now the spectrum of any element $x$ of $e A e$ is the same computed in $B$ or $A$, and is therefore by hypothesis at most countable. Then [9, Lemma 4, p. 375] shows that $B$ is the onedimensional space spanned by $e$. Therefore $e$ is a minimal idempotent of $A$. By [6, Corollary (2.1.9), p. 46] $A e$ and $e A$ are minimal left and right ideals of $A$, respectively.

Finally consider the two-sided ideal of $A, L\left[S_{A}\right]=\left\{a \in A \mid a S_{A}=0\right\}$. If $L\left[S_{A}\right] \neq 0$, then as an algebra, $L\left[S_{A}\right]$ satisfies the hypotheses of the theorem since $A$ does. It follows that $L\left[S_{A}\right]$ has a nonzero minimal left ideal $K$ by the preceding argument. But $K$ is also a minimal left ideal of $A$, and therefore $K^{2}=0$, contradicting the semisimplicity of $A$. Thus $L\left[S_{A}\right]$ must be zero.

THEOREM 2.3. Assume that $A$ is an $A^{*}$-algebra. If the spectrum of every selfadjoint element of $A$ is at most countable, then $A$ has minimal left and right ideals. Also $L\left[S_{A}\right]=0$.

Proof. We may assume that $A$ is not the zero algebra. Let $C$ be a maximal commutative *-subalgebra of $A$, and denote the space of modular maximal ideals of $C$ by $\Omega . C$ is an $A^{*}$-algebra, and hence semisimple (any $A^{*}$-algebra is semisimple by [6, Theorem (4.1.19), p. 188]). For any $w \in C, w=u+i v$ where $u$ and $v$ are some self-adjoint elements of $C$. Clearly the function $w^{\prime}=u^{\prime}+i v^{\prime}$ takes at most a countable number of distinct values on $\Omega$. Then by Theorem $2.1, C$ contains a minimal idempotent $e$, and by [6, Lemma (4.10.1), p. 261], we may assume that $e$ is self-adjoint. Now given a self-adjoint element $v$ of $A$ and $u \in C$, there exists a scalar $\lambda$ such that $e u=u e=\lambda e$, and hence, $(e v e) u=\lambda e v e=u(e v e)$. Also note that eve is self-adjoint. Therefore eve $\in C$, and $e v e$ must be just a scalar multiple of $e$. But every element of $A$ is a linear combination of self-adjoint elements of $A$. Therefore $e$ is a minimal idempotent of $A$, and $A$ has minimal left and right ideals. 
The fact that $L\left[S_{A}\right]=0$ is verified in much the same way as in the proof of Theorem 2.2.

3. An application to $A^{*}$-algebras. In [5], M. A. Naimark asked essentially the following question: If $A$ is an irreducible $B^{*}$-algebra of operators on a separable Hilbert space $\mathscr{H}$ with the property that every nontrivial irreducible representation of $A$ on a Hilbert space is unitarily equivalent to the given representation of $A$ on $\mathscr{H}$, must $A$ be the algebra of all completely continuous operators on $\mathscr{H}$ ? NaImark made progress toward an affirmative answer to this question in [5], and using his results, A. Rosenberg in [7], proved that the question has an affirmative answer. Here using the results of $\S 2$, we prove a variant of the Nalmark-Rosenberg Theorem and at the end of this section, we indicate how their result may be obtained from ours.

TheOREM 3.1. Let $A$ be an irreducible Banach *-subalgebra of the algebra of bounded operators on a separable inner product space $\mathscr{H}$. Assume that every nontrivial irreducible representation of $A$ is equivalent to the given representation of $A$ on $\mathscr{H}$. Then $A$ is a modular annihilator algebra. When $\mathscr{H}$ is a Hilbert space, $A$ is dense in the algebra of all completely continuous operators on $\mathscr{H}$ in the operator norm. If in addition $A$ is a $B^{*}$-algebra, then $A$ is the algebra of all completely continuous operators on $\mathscr{H}$.

Proof. Assume that $v \in A$ and $v=v^{*}$. If $\lambda \in \sigma_{A}(v), \lambda \neq 0$, then either $A(\lambda-v)$ or $A(\lambda-v)$ is a proper modular left ideal of $A$. Choose $\mu$ to be either $\lambda$ or $\lambda$ and such that $A(\mu-v)$ is proper. Then there exists a maximal left ideal $N$ of $A$ such that $A(\mu-v) \subset N$. The left regular representation of $A$ on the quotient space $A-N$ is irreducible. Elements $u \in A$ act on elements $z+N \in A-N$ in the natural way: $u(z+N)=u z+N$. By hypothesis, the representation of $A$ on $A-N$ is equivalent to the given representation of $A$ on $\mathscr{H}$. Therefore there exists an isomorphism $W$ of $A-N$ onto $\mathscr{H}$ such that for every $u, z \in A, u(W(z+N))=W(u z+N)$. Then $(\mu-v)(W(v+N))=W(v(\mu-v)+N)=0$, since $A(\mu-v) \subset N$. Also $W(v+N) \neq 0$. This proves that $\mu$ is an eigenvalue of $v$. Therefore $\mu$ is in the spectrum of $v$ in the algebra of all bounded operators on $\mathscr{H}$, and since $v$ is self-adjoint, $\mu$ is real. Thus $\mu=\lambda=\lambda$. We have shown that when $v \in A$ is self-adjoint, then the nonzero spectrum of $v$ consists entirely of eigenvalues. But then since $\mathscr{H}$ is separable and eigenvectors corresponding to distinct eigenvalues of a self-adjoint operator are orthogonal, it follows that $\sigma_{A}(v)$ is at most countable whenever $v=v^{*}$. By Theorem $2.3 A$ has minimal left and right ideals. Now 0 is a primitive ideal of $A$, and in fact the only primitive ideal of $A$ since all irreducible representations of $A$ are equivalent. Then $A$ is a modular annihilator algebra since $S_{A}$ is contained in no primitive ideal of $A$; see [1, Theorem 4.3(4), p. 570].

Now assume that $\mathscr{H}$ is a Hilbert space. Let $B$ be the closure of $A$ in the operator norm. Then by [2, Lemma 2.6] $S_{B}$ is dense in $B$, and by [8, Lemma 3.11, p. 41], 
this implies that $B$ is a modular annihilator $B^{*}$-algebra of operators. It remains to be shown that an irreducible modular annihilator $B^{*}$-algebra of operators on a Hilbert space $\mathscr{H}$ is the algebra of completely continuous operators on $\mathscr{H}$. But by $[8$, Theorem $4.1, \mathrm{p} .42]$ such an algebra must be dual and then the result follows by [6, Corollary (4.10.20), p. 269] and [6, Theorem (4.10.22), p. 270].

Now the combination of $[6$, Lemma (4.9.11), p. 254] and [6, Theorem (4.9.8), p. $251]$ implies that any irreducible representation of a $B^{*}$-algebra is algebraically equivalent to an irreducible *-representation of the algebra on a Hilbert space. Thus if all the nontrivial irreducible *-representations on a Hilbert space of a $B^{*}$-algebra $A$ are unitarily equivalent (or in fact merely algebraically equivalent), then all nontrivial irreducible representations of $A$ are algebraically equivalent. These remarks show that the Theorem of NaImark and Rosenberg follows from Theorem 3.1.

4. Modular annihilator algebras. It was shown in [3] that if $A$ is a semisimple normed modular annihilator algebra, then $A$ has the property that the spectrum of every element has no nonzero limit point; see [3], the Corollary to Theorem 3.4. In this section we prove the converse of this theorem with the assumption that $A$ is a Banach algebra. We obtain the result for the case where $A$ is commutative first, and then extend this special case to the general theorem.

THEOREM 4.1. Let $A$ be a semisimple commutative Banach algebra with the property that for every $u \in A, \sigma_{A}(u)$ has no nonzero limit points. Then $A$ is a modular annihilator algebra.

Proof. We may assume that $A$ is not finite dimensional (any semisimple finite dimensional algebra is a modular annihilator algebra). Let $\Omega$ be the space of modular maximal ideals of $A$, and let $\Gamma$ be the set of isolated points of $\Omega$. By Theorem 2.1, $\Gamma$ is nonempty. First we prove that $\Gamma$ is a closed set in $\Omega$. Assume that $\phi \in \Omega$ is a limit point of $\Gamma$. Choose $v \in A$ such that $v^{\prime}(\phi)=1$. Now 1 is an isolated point of $\sigma_{A}(v)$, so we may choose an open subset of the complex plane $U$ such that $U \cap \sigma_{A}(v)=\{1\}$. Then the set $N=\left\{\psi \in \Omega \mid v^{\prime}(\psi)=1\right\}$ is an open subset of $\Omega$ which contains $\phi$. But then $N$ must contain an infinite number of points of $\Gamma$. We choose a sequence $\left\{\phi_{k}\right\}$ of distinct points in $\Gamma \cap N$. There exists a corresponding sequence of minimal idempotents $\left\{e_{k}\right\}$ in $A$ with the property that $e_{k}^{\prime}\left(\phi_{k}\right)=1, e_{k}^{\prime}(\psi)=0, \psi \in \Omega$, $\psi \neq \phi_{k}$. Finally we choose a sequence of distinct complex numbers $\left\{\lambda_{k}\right\}$ with the property that $\left|\lambda_{k}\right| \leqq 1 /\left\|e_{k}\right\| 2^{k}$. Set $w=v+\sum_{k=1}^{\infty} \lambda_{k} e_{k}$. Then $w^{\prime}\left(\phi_{k}\right)=1+\lambda_{k}$ and $\lambda_{k} \rightarrow 0$ as $k \rightarrow \infty$. This is a contradiction, since 1 must be an isolated point of $\sigma_{A}(w)$. Therefore $\Gamma$ must have no limit points in $\Omega$, and in particular $\Gamma$ is a closed set.

Now let $\Delta=\Omega-\Gamma . \Delta$ is hull-kernel closed since $\Delta$ is the intersection of the sets $\left\{\psi \in \Omega \mid e^{\prime}(\psi)=0\right\}$ for all minimal idempotents $e \in A$. By $k(\Delta)$ we mean as usual the intersection of all the ideals in $\Delta$. Let $B$ be the quotient algebra $A / k(\Delta)$. Then $B$ is semisimple and the spectrum of any element in $B$ has no nonzero limit points. By Theorem 2.1, if $B$ is nonzero then $B$ must have a nonzero minimal ideal. But by 
[6, Theorem (3.1.17), p. 116] $\Delta$ is homeomorphic to the maximal ideal space of $B$, and hence $\Delta$ must contain isolated points. Since $\Gamma$ is closed, $\Delta$ is open, and then any isolated point of $\Delta$ is an isolated point of $\Omega$. This contradiction proves that in fact $B$ is the zero algebra and $\Delta$ must be empty. We have established that $\Gamma=\Omega$.

Suppose that $M$ is any maximal modular ideal of $A$. Since $M$ is an isolated point of $\Omega$, there exists a minimal idempotent $e \in A$ such that $e^{\prime}(M)=1, e^{\prime}(N)=0$ if $N \in \Omega, N \neq M$. But then whenever $u \in M,(u e)^{\prime}(N)=0$ for every $N \in \Omega$. Since $A$ is semisimple, we conclude that $M e=0$. Thus $A$ is a modular annihilator algebra by definition.

THEOREM 4.2. Let $A$ be any complex semisimple Banach algebra. $A$ is a modular annihilator algebra if and only if for any $u \in A, \sigma_{A}(u)$ has no nonzero limit points.

Proof. As we mentioned previously the "only if" part of this theorem follows from [3], the Corollary to Theorem 3.4. Thus we assume that for every $u \in A, \sigma_{A}(u)$ has no nonzero limit points. Then by Theorem $2.2, L\left[S_{A}\right]=0$. This implies that every nonzero right ideal of $A$ contains a minimal right ideal of $A$ (and hence a minimal idempotent of $A$ ); see [8, Lemma 3.1, p. 37].

Assume that $e$ is a nonzero idempotent of $A$. Let $\mathscr{M}$ be a maximal orthogonal set of minimal idempotents of $A$ in $e A$. If $\mathscr{M}$ is infinite, we can choose a sequence of distinct idempotents $\left\{e_{k}\right\} \subset \mathscr{M}$. Then choose $\left\{\lambda_{k}\right\}$ a sequence of distinct complex numbers with the property that $\left|\lambda_{k}\right| \leqq 1 /\left\|e_{k}\right\| 2^{k}$. Finally set $w=e+\sum_{k=1}^{\infty} \lambda_{k} e_{k}$. Each $e_{k} \in e A$, and thus $e_{k}=e e_{k}$. Then $w e_{k}=\left(1+\lambda_{k}\right) e_{k}$ for each $k$. Therefore the sequence $\left\{1+\lambda_{k}\right\}$ must be in the spectrum of $w$, a contradiction. It follows that $\mathscr{M}$ must be finite. Then by an argument similar to the proof of Theorem 2.2 of [3], it can be shown that $e \in S_{A}$. We have proved that every idempotent of $A$ is in $S_{A}$.

Now we prove directly that $A$ is a modular annihilator algebra. Let $M$ be a maximal modular left ideal of $A$. By [8, Lemma 3.3, p. 38] either $S_{A} \subset M$ or $R[M]$ $\neq 0$ (where $R[M]=\{a \in A \mid M a=0\}$ ). Suppose that $S_{A} \subset M$. Since $M$ is modular, there exists $u \in A$ such that $A(1-u) \subset M$. Let $C$ be a maximal commutative subalgebra of $A$ containing $u$. Let $\Omega$ be the space of modular maximal ideals of $C$. For every $v \in C, \sigma_{A}(v)=\sigma_{C}(v)$. Thus by Theorem $4.1, C / \operatorname{rad}(C)$ is a modular annihilator algebra. Denote $C / \mathrm{rad}(C)$ by $B$, and let $\pi$ be the natural homomorphism of $C$ onto $B$. Since $B$ is a semisimple modular annihilator algebra, $\pi(u)$ is quasi-regular modulo $S_{B}$ by [8, Theorem $3.4(3)$, p. 38]. Therefore there exist elements $x_{1}, x_{2}, \ldots, x_{n}$ and $w$ in $C$ and minimal idempotents $f_{1}, f_{2}, \ldots, f_{n}$ in $B$ such that $\pi(w)(1-\pi(u))+\pi(u)$ $=\pi\left(x_{1}\right) f_{1}+\cdots+\pi\left(x_{n}\right) f_{n}$. Now by [6, Theorem (2.3.9), p. 58], there are idempotents $e_{k} \in C$ such that $\pi\left(e_{k}\right)=f_{k}, 1 \leqq k \leqq n$. By the definition of $\pi$, there exists $r \in \operatorname{rad}(C)$ such that $r=w(1-u)+u-\left(x_{1} e_{1}+\cdots+x_{n} e_{n}\right)$. Then $A(1-r) \subset A(1-w)(1-u)$ $+A\left(x_{1} e_{1}+\cdots+x_{n} e_{n}\right)$. But $A(1-w)(1-u) \subset M$, and $A\left(x_{1} e_{1}+\cdots+x_{n} e_{n}\right) \subset S_{A} \subset M$ (recall that we have shown that every idempotent in $A$ is in $S_{A}$ ). Therefore $A(1-r)$ $\subset M$, and this contradicts the fact that $\{0\}=\sigma_{C}(r)=\sigma_{A}(r)$. Hence $R[M] \neq 0$, and $A$ must be a modular annihilator algebra. 
COROllary. Assume $A$ is a semisimple complex modular annihilator Banach algebra. Then if $B$ is any closed subalgebra of $A$, then $B / \operatorname{rad}(B)$ is a modular annihilator algebra.

Proof. It is sufficient to note that $\sigma_{B}(u) \cup\{0\}=\sigma_{A}(u) \cup\{0\}$ for all $u \in B$ by [6, Theorem (1.6.12), p. 33]. Then apply Theorem 4.2.

\section{REFERENCES}

1. B. A. Barnes, Modular annihilator algebras, Canad. J. Math. 18 (1966), 566-578.

2. —_, Algebras with the spectral expansion property, Illinois J. Math. 11 (1967), 284-290.

3. - A generalized Fredholm theory for certain maps in the regular representations of an algebra, Canada. J. Math. (to appear).

4. E. Hille and R. S. Phillips, Functional analysis and semi-groups, rev. ed., Amer. Math. Soc. Colloq. Publ., Vol. 31, Amer. Math. Soc., Providence, R. I., 1957.

5. M. A. Nalmark, On a problem of the theory of rings with involution, Uspehi Mat. Nauk 6 (1951), 160-164.

6. C. E. Rickart, Banach algebras, Princeton Univ. Press, Princeton, N. J., 1960.

7. A. Rosenberg, The number of irreducible representations of simple rings with no minimal ideals, Amer. J. Math. 75 (1953), 523-530.

8. B. Yood, Ideals in topological rings, Canad. J. Math. 16 (1964), 28-45.

9. I. Kaplansky, Ring isomorphisms of Banach algebras, Canad. J. Math. 6 (1954), 374-381.

UNIVERSTTY OF OREGON,

EUGENE, OREGON 